\title{
Musculofascial lengthening for the treatment of patients with medial epicondylitis and coexistent ulnar neuropathy
}

\author{
H. S. Gong, \\ M. S. Chung, \\ E. S. Kang, \\ J. H. Oh, \\ Y. H. Lee, \\ G. H. Baek
}

From Seoul National University Bundang Hospital, Seongnam, Korea

The outcome of surgery in patients with medial epicondylitis of the elbow is less favourable in those with co-existent symptoms from the ulnar nerve. We wanted to know whether we could successfully treat such patients by using musculofascial lengthening of the flexorpronator origin with simultaneous deep transposition of the ulnar nerve. We retrospectively reviewed 19 patients who were treated in this way. Seven had grade I and 12 had grade lla ulnar neuropathy. At a mean follow-up of 38 months (24 to 48), the mean visual analogue scale pain scores improved from 3.7 to 0.3 at rest, from 6.6 to 2.1 with activities of daily living, and from $\mathbf{7 . 9}$ to $\mathbf{2 . 3}$ at work or sports, and the mean disabilities of the arm, shoulder and hand scores improved from 42.2 to 23.5 .

These results suggest that this technique can be effective in treating patients with medial epicondylitis and coexistent ulnar nerve symptoms.

The primary cause of medial epicondylitis is said to be overuse of the flexor-pronator muscles, most commonly the pronator teres and the flexor carpi radialis, which contain pathological degenerative changes in the tendon by which they arise from the medial epicondyle. ${ }^{1-3}$

Various operations have been described for elbows in which conservative treatment has been unsuccessful, including percutaneous release, ${ }^{4}$ debridement and reattachment, ${ }^{1,5}$ resection of the angiofibroblastic tissue and drilling, ${ }^{6}$ incision and debridement of the involved muscle origin without reattachment, ${ }^{1}$ and division of the whole flexor origin without reattachment or debridement. ${ }^{7}$ As ulnar nerve symptoms are associated with medial epicondylitis in $23 \%$ to $60 \%$ of patients, ${ }^{1,5-7}$ ulnar nerve release or transposition is combined when indicated. Although most studies have reported good to excellent results in $86 \%$ to $97 \%$ of patients, ${ }^{1,5,6}$ results appear to be less favourable for the elbows with co-existent ulnar nerve symptoms. ${ }^{1,3,7}$ McCluskey $^{3}$ noted that results are poorer when ulnar nerve symptoms are more severe and better when they are mild or absent. ${ }^{3}$ Gabel and Morrey ${ }^{1}$ reported good results in only two of five elbows that had moderate or severe associated ulnar neuropathy compared with 24 of 25 elbows with no or mild ulnar neuropathy. Kurvers and Verhaar ${ }^{7}$ found that only three of 24 elbows with co-existent ulnar neuritis were symptomfree after operation.
The musculofascial lengthening technique with submuscular transposition of the ulnar nerve is a modification of the original submuscular transposition method $^{8}$ for relieving ulnar nerve compression at the elbow. ${ }^{9} \mathrm{We}$ thought this procedure would also have a tension-releasing and stress relieving effect on the insertion of the flexor-pronator muscles and, in addition, would allow immediate movement and nerve gliding. Furthermore, exact identification of the pathological tissue is not required in this procedure since the whole musculotendinous mass is released; thus, a more consistent procedure can be performed because clear identification of the pathological tissue is seldom made. We have therefore reviewed the results of flexor muscle $Z$ plasty release and ulnar nerve transposition to find whether it was an effective treatment for medial epicondylitis of the elbow with concomitant ulnar nerve symptoms.

\section{Patients and Methods}

We retrospectively reviewed 19 patients who had musculofascial lengthening of the flexorpronator muscles and submuscular ulnar nerve transposition for the treatment of medial epicondylitis associated with ulnar nerve symptoms between May 2003 and February 2007. All patients had local tenderness over the origin of the flexor-pronator muscles near the medial epicondyle and pain provoked by resisted palmar flexion of the wrist, which was 
Table I. Modified McGowan grading system for ulnar neuropathy ${ }^{11}$

\begin{tabular}{ll}
\hline Grade & Description \\
\hline $\mathrm{I}$ & $\begin{array}{l}\text { Patients with subjective symptoms but without } \\
\text { objective findings }\end{array}$ \\
Ila & $\begin{array}{l}\text { Patients with good intrinsic strength }(4 / 5) \text { and } \\
\text { without intrinsic atrophy }\end{array}$ \\
IIb & $\begin{array}{l}\text { Patients with fair intrinsic strength (3/5) and with } \\
\text { intrinsic atrophy }\end{array}$ \\
III & $\begin{array}{l}\text { Patients with marked intrinsic atrophy and } \\
\text { sensory disturbance }\end{array}$ \\
\hline
\end{tabular}

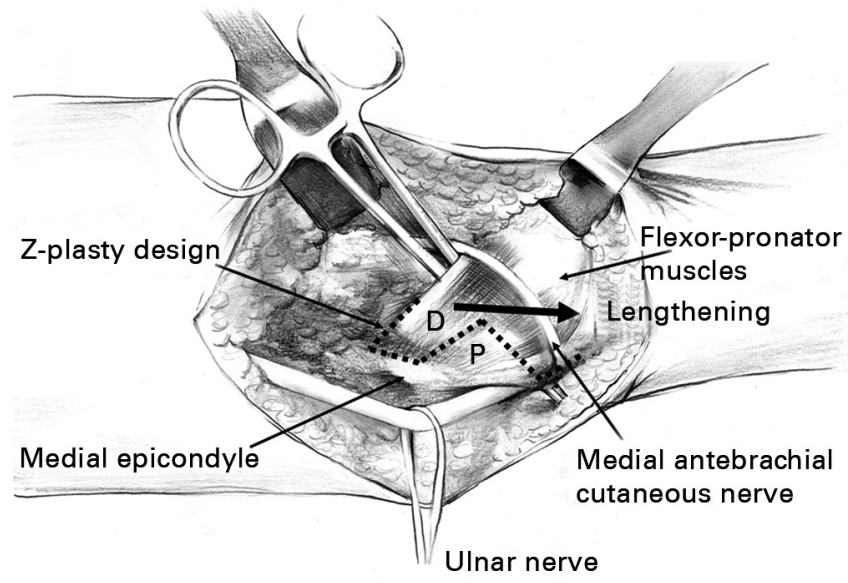

Fig. 1

A drawing showing the Z-plasty design of the musculofascial lengthening of flexor-pronator muscles in the left elbow (dotted line). The hand is to the right. The ulnar nerve is released (sling) and a haemostat is placed under the flexor-pronator muscles with a Z-plasty design marked on them. The proximal flap $(P)$ contains the flexor carpi ulnaris and the distal flap (D) contains the pronator teres and common flexor tendons. The thick arrow indicates the lengthening direction.

resistant to conservative treatment for more than one year. All also had altered sensation affecting the ulnar aspect of the hand and at least one positive test for the ulnar neuropathy, such as Tinel's sign at the elbow or the elbow flexion test, and a minimum follow-up of two years. We excluded patients who had medial epicondylitis without ulnar nerve symptoms, primary ulnar neuropathy at the elbow with minimal elbow pain or previous ulnar nerve surgery, ulnar ligament insufficiency, osteoarthritis or inflammatory arthritis of the elbow, and major limb trauma or reflex sympathetic dystrophy.

There were four men and 15 women with a mean age at the time of operation of 51 years (29 to 77). The mean duration of symptoms before the operation was 37 months (14 to 84). The dominant elbow was involved in seven patients, non-dominant in ten, and both in two. These two patients had staged operations of which the first, on the dominant side, was included in this study. There was a preceding history of golf in seven, and occupational overuse in one, but no specific provocative activity in the remaining
11 patients. The mean follow-up was for 38 months (24 to 58). No patients were lost to follow-up. Institutional review board approval was obtained for this study.

Pre-operative radiographs showed calcification in the region of the medial epicondyle in five elbows. The patients received a mean number of 3.5 (1 to 10) injections to the elbow before referral. Electrodiagnostic studies confirmed ulnar neuropathy in six patients, and the others had symptoms and signs of ulnar nerve involvement with normal electrodiagnostic tests. There were seven patients with sensory symptoms such as tingling and paraesthesie and 12 had abnormal two-point discrimination. No patient had atrophy of the intrinsic muscles or a positive Froment or Waternberg sign. ${ }^{10}$ We classified all patients using a modified McGowan grading system (Table I). ${ }^{11}$ Seven patients were grade I and 12 were grade IIa. All electrodiagnostically confirmed patients had McGowan grade IIa.

Operative procedure and post-operative care. All operations were performed by one surgeon (HSG), using the technique described by Dellon and Coert. ${ }^{9}$ In brief, a $10 \mathrm{~cm}$ to $12 \mathrm{~cm}$ incision is made on the posteromedial aspect of the elbow. The medial antebrachial cutaneous nerve is identified and protected. The cubital tunnel is opened and dissection continued distally to divide the fascia between the two heads of flexor carpi ulnaris. The ulnar nerve is released and the medial intermuscular septum excised. All patients were thought to have compression distal to the epicondyle where the nerve passes between the heads of the flexor carpi ulnaris. The musculofascial lengthening is made in a ' $z$ ' fashion in the flexor-pronator muscles (Figs 1 and 2a). The proximal flap, containing the flexor carpi ulnaris, is left attached to the medial epicondyle and the distal flap, containing the pronator teres and common flexor tendons, slides distally (Fig. 2b). The ulnar nerve is moved forward to lie on the brachialis muscle, and the proximal and distal flaps are joined with three horizontal mattress sutures (Fig. 2c).

Post-operatively, the elbow is supported in a sling for two weeks, and immediate passive movement encouraged to facilitate nerve gliding and to reduce the formation of adhesions. After two weeks, active range of movement is instituted, allowing progression to light daily activities, and from six weeks strengthening exercises. Return to sport is allowed three months after operation.

Outcome assessment. All pre-operative data were obtained three or four weeks prior to surgery and included visual analogue scale (VAS) pain ratings, grip strength measurement, and the Disabilities of the Arm, Shoulder and Hand (DASH) score. ${ }^{12}$ Final post-operative review included these three assessments, and in addition, the modified Wilson and Krout score for ulnar neuropathy, ${ }^{13}$ which is specifically designed to assess post-operative changes, the grading system of Nirschl and Pettrone, ${ }^{14}$ and overall satisfaction VAS ratings. For VAS pain ratings, a scale was used between 0 (no pain) to 10 (severe pain) at rest, with activities of daily living and at work or during sports activities. Similarly, patients were asked to indicate their 


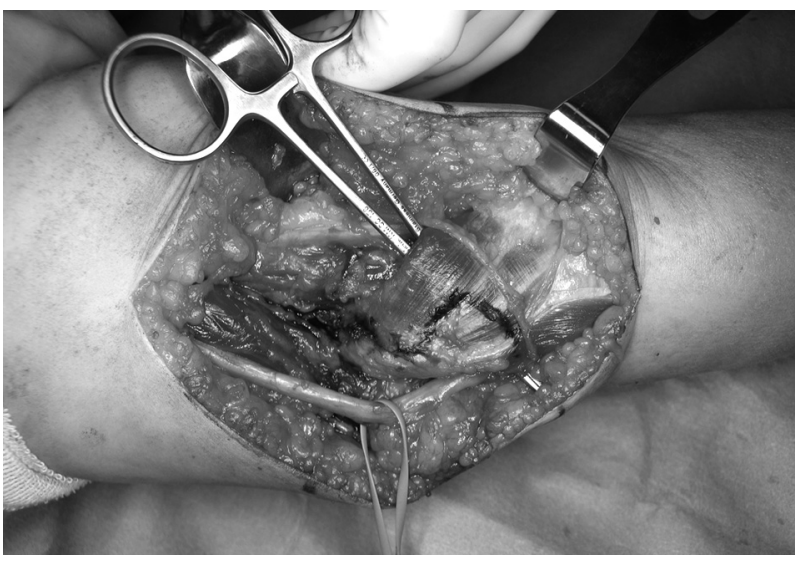

Fig. 2a

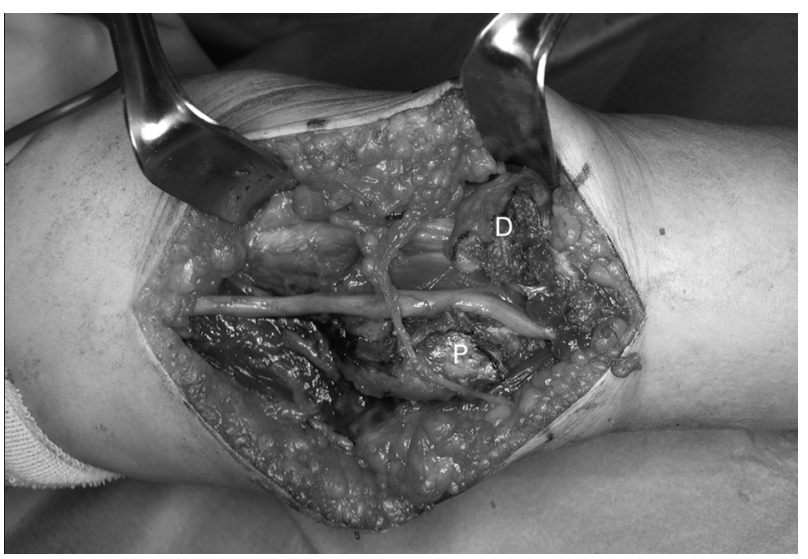

Fig. 2b

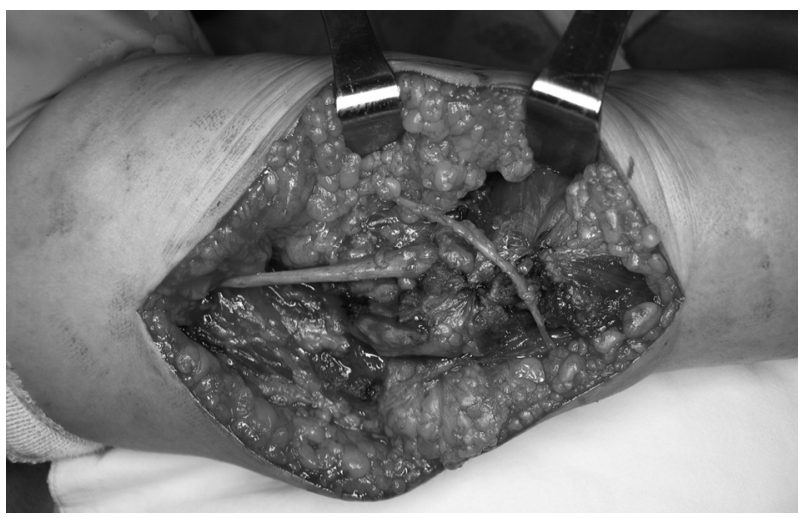

Fig. 2c

Photographs showing the operative procedure with a) the hand to the right. The ulnar nerve is released (sling) and a haemostat is placed under the flexor-pronator muscles with a z-plasty design marked on them, b) the flexor-pronator muscles are divided, and the proximal flap (P), containing the flexor carpi ulnaris, is left attached to the medial epicondyle and the distal flap (D), containing the pronator teres and common flexor tendons, slides distally. The ulnar nerve is transposed anteriorly and placed on the brachialis muscle and c) after obtaining haemostasis, the proximal and distal flaps are sutured with three horizontal mattress sutures. The median antebrachial cutaneous nerve is seen over the repaired flexor-pronator muscles.

post-operative satisfaction by scoring from 0 (complete dissatisfaction) to 10 (complete satisfaction). Grip strength was measured with the Jamar hand dynamometer (Asimow
Engineering, Los Angeles, California) in a standardised manner. In a neutral position of the arm, forearm and wrist, three consecutive attempts with intervals of one minute were each measured in kilograms. The DASH score quantifies general disabilities related to the arm and is scaled between 0 and 100, where higher scores represent more disability. ${ }^{12}$ In the modified Wilson and Krout grading system, ${ }^{13}$ the scale is excellent, minimal motor and sensory changes and no tenderness at the incision site; good, occasional ache and mild sensory or motor changes; fair, improved but persistent sensory or motor changes; and poor, no improvement or the condition worsening. The grading system of Nirschl and Pettrone ${ }^{14}$ was excellent, full return to all activity with no pain; good, full return to all activity with occasional mild pain; fair, normal activity with no pain, substantial pain with heavy activity, and $75 \%$ or better overall improvement; and failure, no relief of preoperative symptoms. All assessments were made by a physician's assistant who was not directly involved in the care of the patients.

Statistical analysis. The differences between the preoperative VAS pain, grip strength, and DASH scores and those at final follow-up were analysed using the Wilcoxon signed-rank test. Comparisons of satisfaction scores between subgroups according to pre-operative variables were performed using the Mann-Whitney test. Statistical significance was considered to be $\mathrm{p}<0.05$. The relationship between the number of pre-operative steroid injections and post-operative satisfaction was analysed by using nonparametric bivariate correlation analysis.

\section{Results}

All VAS pain scores improved after operation. The mean VAS pain score improved from 3.7 to 0.3 at rest $(\mathrm{p}<0.001)$, from 6.6 to 2.1 with activities of daily living ( $<<0.001$ ), and from 7.9 to 2.3 at work or sports $(\mathrm{p}<0.001)$. The mean grip strength improved from $18.6 \mathrm{~kg}$ to $22.8 \mathrm{~kg}$ ( $\mathrm{p}<0.001)$. The increase in the mean strength of the uninvolved side $(23.9 \mathrm{~kg}$ pre-operatively and $24.3 \mathrm{~kg}$ after operation) was not significant $(\mathrm{p}=0.097)$. The mean DASH score improved from 42.2 points to 23.5 points $(\mathrm{p}<0.001)$ (Table II). The mean satisfaction VAS score was 8.4 (5 to 10 ). There was no relationship between the satisfaction score and other variables such as electrodiagnostic studies, calcification on radiographs, and grading of ulnar neuropathy. However, the correlation between the number of pre-operative steroid injections and post-operative satisfaction was significant with Spearman's coefficient of $0.564(\mathrm{p}=0.012)$, indicating that those who had received more injections had a better post-operative result.

Using the modified Wilson and Krout grading system for ulnar neuropathy, ${ }^{12}$ four patients were rated 'excellent' and 15 'good'. All of those who were rated 'good' had no sensory or motor deficit but still had some tenderness at the incision site. Using the Nirschl and Pettrone grading system, ${ }^{14}$ six patients had an excellent result, ten good, and three fair. 
Table II. Summary of major outcome variables (mean; range)

\begin{tabular}{|c|c|c|c|}
\hline Variable* & Pre-operative & Post-operative & p-value ${ }^{\dagger}$ \\
\hline \multicolumn{4}{|l|}{ VAS pain score (0 to 10$)$} \\
\hline At rest & 3.7 (2 to 5$)$ & 0.3 (0 to 2 ) & $<0.001$ \\
\hline With activities of daily living & 6.6 (4 to 9$)$ & 2.1 ( 1 to 4$)$ & $<0.001$ \\
\hline At work or sports & $7.9(7$ to 10$)$ & 2.3 (1 to 4$)$ & $<0.001$ \\
\hline \multicolumn{4}{|l|}{ Grip strength (kg) } \\
\hline Operation side & 18.6 (9.1 to 37.3$)$ & $22.8(12.7$ to 47.3$)$ & $<0.001$ \\
\hline Opposite side & $23.9(12.7$ to 41.8$)$ & $24.3(14.5$ to 46.4$)$ & 0.097 \\
\hline DASH scores (0 to 100$)$ & 42.2 (27 to 59 ) & 23.5 (12 to 49$)$ & $<0.001$ \\
\hline
\end{tabular}

There were no major complications such as infection or joint contracture. However, two patients had mild numbness on the posteromedial side of the elbow. The two patients with bilateral epicondylitis also chose to have the operation on the opposite side.

\section{Discussion}

Our study demonstrates that musculofascial lengthening of the flexor pronator muscles at the elbow combined with anterior transposition of the ulnar nerve gives good results in patients with coexistent medial epicondylitis and ulnar nerve symptoms. Different operative techniques have been proposed to deal with the ulnar nerve under these circumstances. Gabel and Morrey ${ }^{1}$ preferred submuscular transposition of the nerve with elevation and debridement of the entire flexor muscles when there was focal compression of the nerve but stated that treatment of the epicondylitis alone is sufficient in cases with mild ulnar neuropathy since resolution of the inflammation should alleviate the neuropathy unless there is focal compression in the distal part of the cubital tunnel. In their study, however, only five of 16 patients with ulnar neuropathy improved without ulnar nerve release and others had ulnar nerve surgery. Kurvers and $\operatorname{Verhaar}^{7}$ reported that in patients with co-existent ulnar neuropathy the results of release of the flexor attachment alone or simple release of the retinaculum over the cubital tunnel alone were unsatisfactory. They suspected that degenerative changes in patients with medial epicondylitis are not limited to the origin of the flexor muscles but may involve the peritendinous tissues as well, resulting in oedema and sclerosis, and concluded that in situ ulnar nerve decompression which maintains the nerve within the vicinity of the degenerative and inflamed soft tissues at the medial epicondyle is ineffective in treating neuropathy. In their study, symptoms of neuropathy persisted in 15 of 22 patients who had in situ decompression (17 patients) or no release (five patients). Vangsness and Jobe ${ }^{5}$ performed anterior submuscular transposition of the ulnar nerve in addition to debridement and re-attachment of the flexorpronator muscles in four of five elbows with concomitant ulnar neuropathy. They directly re-attached the flexorpronator muscles, while our technique involved a $\mathrm{Z}$ length- ening of the muscles. Although they reported excellent results, they had placed the arm in a $90^{\circ}$ posterior splint and had the patients avoid resisted wrist flexion or pronation for six to eight weeks after operation. We performed submuscular transposition in all patients with ulnar nerve symptoms, following the advice of Kurvers and Verhaar ${ }^{7}$ who reported that leaving the ulnar nerve near the degenerative and inflamed tissue at the medial epicondyle gives poor results.

The advantage of the musculofascial lengthening technique is that it can create space for the ulnar nerve, permit reconstruction of the flexor-pronator muscle mass without tension and allow immediate post-operative flexion and extension of the elbow and gliding of the nerve, which can prevent the perineural fibrosis that occurs if mobilisation is delayed. ${ }^{15}$ Another advantage is that exact identification and removal of any pathological tissue is not required as the whole muscle insertion is released; thus, a more consistent procedure can be performed. Resection of the angiofibroblastic tissue alone requires exact identification of the degenerative tissue, which is usually located deep, and there is a potential risk of leaving pathological tissue, which can be a source of residual pain as suggested by a study on lateral epicondylitis. ${ }^{16}$ Furthermore, caution is required when undertaking deep debridement of the flexor-pronator origin posterior to the accessory anterior oblique ligament since damage to this ligament may result in iatrogenic valgus instability. ${ }^{17}$ However, our procedure has the disadvantages of a relatively large scar and a technically more complex procedure. In addition, although our patients had improvements of grip strength, there may be a decrease in muscle power resulting from the Z-lengthening; Nouhan and Kleinert ${ }^{18}$ noted a $10 \%$ decrease in grip strength in patients who had normal pre-operative power after musculofascial lengthening of flexor-pronator muscles for cubital tunnel syndrome. The effect of the lengthening on the muscle kinematics may need further evaluation.

There are several limitations to this study. Firstly, we did not include patients with severe ulnar neuropathy and weakness of the intrinsic muscles, and only six patients had positive electrodiagnostic tests, which may raise concerns on the justification for transposition in those with normal 
nerve conduction studies. However, patients in the previous studies regarding medial epicondylitis and ulnar nerve involvement had characteristics similar to our patients and their electrodiagnostic tests were mostly normal. ${ }^{1,3,7}$ In the study by Gabel and Morrey, ${ }^{1}$ there were 11 mild, four moderate and one severe case of ulnar neuropathy, and there was no comment on electrodiagnostic tests. In the study by Kurvers and Verhaar, ${ }^{7}$ which reported the least favourable outcomes in patients with an ulnar neuropathy, no patients had positive electrodiagnostic tests at the elbow. Secondly, our study population was small and composed of more women than men, more nondominant than dominant arms, and few sports-related injuries, which may not represent the prevalence characteristics of medial epicondylitis. Thirdly, our study did not have a control group and the outcome tools used in the previous literature were various. Therefore, it was difficult to compare directly the results of our method with those of the previous techniques, although we tried to include as many available outcome measurements as possible. Prospective, comparative studies will be necessary to demonstrate clearly which procedure is best for patients with medial epicondylitis and coexistent ulnar nerve symptoms. Given the limitations mentioned, our data suggest that the musculofascial lengthening technique with ulnar nerve transposition can be a reliable form of treatment under these circumstances.

\section{Supplementary material for the web}

ë A table showing the comparison of surgical treatment of medial epicondylitis and ulnar nerve symptoms is available with the electronic version of this article on our website at www.jbjs.org.uk.

The authors thank Ms. Y.-H. Kim for her assistance during the data collection. No benefits in any form have been received or will be received from a commercial party related directly or indirectly to the subject of this article.

\section{References}

1. Gabel GT, Morrey BF. Operative treatment of medial epicondylitis: influence of concomitant ulnar neuropathy at the elbow. J Bone Joint Surg [Am] 1995;77-A:1065-9.

2. Ciccotti MG, Ramani MN. Medial epicondylitis. Tech Hand Up Extrem Surg 2003; 7:190-6.

3. McCluskey GM. Open treatment of medial epicondylitis. In: Yamaguchi K, King GJW, McKee MD, O'Driscoll SWM, eds. Advanced reconstruction: elbow. Rosemont: American Academy of Orthopaedic Surgeons, 2007:137-42.

4. Baumgard SH, Schwartz DR. Percutaneous release of the epicondylar muscles for humeral epicondylitis. Am J Sports Med 1982;10:233-6.

5. Vangsness CT Jr, Jobe FW. Surgical treatment of medial epicondylitis: results in 35 elbows. J Bone Joint Surg [Br] 1991;73-B:409-11.

6. Ollivierre CO, Nirschl RP, Pettrone FA. Resection and repair for medial tennis elbow: a prospective analysis. Am J Sports Med 1995;23:214-21.

7. Kurvers $\mathbf{H}$, Verhaar $\mathbf{J}$. The results of operative treatment of medial epicondylitis. $J$ Bone Joint Surg [Am] 1995;77-A:1374-9.

8. Learmonth JR. Technique for transplanting the ulnar nerve. Surg Gynecol Obstet 1942;75:792-3.

9. Dellon AL, Coert JH. Results of the musculofascial lengthening technique for submuscular transposition of the ulnar nerve at the elbow. J Bone Joint Surg [Am] 2003;85-A:1314-20.

10. Goldman SB, Brininger TL, Schrader JW, Koceja DM. A review of clinical tests and signs for the assessment of ulnar neuropathy. J Hand Ther 2009;22:209-19.

11. Goldberg BJ, Light TR, Blair SJ. Ulnar neuropathy at the elbow: results of medial epicondylectomy. J Hand Surg Am 1989;14:182-8.

12. Hudak PL, Amadio PC, Bombardier C. Development of an upper extremity outcome measure: the DASH (disabilities of the arm, shoulder and hand) [corrected]. The Upper Extremity Collaborative Group (UECG). Am J Ind Med 1996;29:602-8.

13. Wilson DH, Krout R. Surgery of ulnar neuropathy at the elbow: 16 cases treated by decompression without transposition: technical note. J Neurosurg 1973;38:780-5.

14. Nirschl RP, Pettrone FA. Tennis elbow: the surgical treatment of lateral epicondylitis. J Bone Joint Surg [Am] 1979;61-A:832-9.

15. Williams EH, Dellon AL. Anterior submuscular transposition. Hand Clin 2007;23:345-58

16. Cummins CA. Lateral epicondylitis: in vivo assessment of arthroscopic debridement and correlation with patient outcomes. Am J Sports Med 2006;34:1486-91.

17. McCluskey GM 3rd, Merkley MS. Lateral and medial epicondylitis. In: Baker CL Jr, Plancher K, eds. Operative treatment of elbow injuries. New York: Springer-Verlag, 2002:79-88.

18. Nouhan R, Kleinert JM. Ulnar nerve decompression by transposing the nerve and Zlengthening the flexor-pronator mass: clinical outcome. J Hand Surg [Am] 1997;22:127-31 